\title{
Strongly relatively nonexpansive sequences generated by firmly nonexpansive-like mappings
}

Koji Aoyama ${ }^{*}$ and Fumiaki Kohsaka²

"Correspondence:

aoyama@le.chiba-u.ac.jp

'Department of Economics, Chiba

University, Yayoi-cho, Inage-ku,

Chiba-shi, Chiba, 263-8522, Japan

Full list of author information is

available at the end of the article

\begin{abstract}
We show that a strongly relatively nonexpansive sequence of mappings can be constructed from a given sequence of firmly nonexpansive-like mappings in a Banach space. Using this result, we study the problem of approximating common fixed points of such a sequence of mappings.

MSC: Primary 47H09; secondary 47H05; 65J15

Keywords: Banach space; firmly nonexpansive-like mapping; firmly nonexpansive mapping; fixed point; mapping of type (P); proximal point algorithm; uniform convexity constant
\end{abstract}

\section{Introduction}

The aim of the present paper is twofold. Firstly, we construct a strongly relatively nonexpansive sequence from a given sequence of firmly nonexpansive-like mappings with a common fixed point in Banach spaces. Secondly, we obtain two convergence theorems for firmly nonexpansive-like mappings in Banach spaces and discuss their applications.

The class of firmly nonexpansive-like mappings (or mappings of type (P)) introduced in [1] plays an important role in nonlinear analysis and optimization. In fact, the fixed point theory for such mappings can be applied to several nonlinear problems such as zero point problems for monotone operators, convex feasibility problems, convex minimization problems, equilibrium problems, and so on; see [1-3] and Section 5 for more details.

Let $C$ be a nonempty closed convex subset of a smooth, strictly convex, and reflexive Banach space $X, J$ the normalized duality mapping of $X$ into $X^{*}$, and $T: C \rightarrow X$ a firmly nonexpansive-like mapping; see (2.16). The set of all fixed points of $T$ is denoted by $F(T)$. It is known [1, Theorem 7.4] that if $C$ is bounded, then $\mathrm{F}(T)$ is nonempty. Martinet's theorem [4, Théorème 1] ensures that if $X$ is a Hilbert space and $C$ is bounded, then the sequence $\left\{T^{n} x\right\}$ converges weakly to an element of $\mathrm{F}(T)$ for each $x \in C$. However, we do not know whether Martinet's theorem holds for firmly nonexpansive-like mappings in Banach spaces.

On the other hand, using the metric projections in Banach spaces, Kimura and Nakajo [5, Theorems 6 and 7] recently obtained generalizations of the results due to Crombez [6, Theorem 3] and Brègman [7, Theorem 1].

O2014 Aoyama and Kohsaka; licensee Springer. This is an Open Access article distributed under the terms of the Creative Commons Attribution License (http://creativecommons.org/licenses/by/2.0), which permits unrestricted use, distribution, and reproduction in any medium, provided the original work is properly cited. 
In this paper, inspired by [5], we investigate the asymptotic behavior of the following sequences $\left\{x_{n}\right\}$ and $\left\{y_{n}\right\}$ in a uniformly smooth and 2-uniformly convex Banach space $X$ :

$$
x_{n+1}=Q_{C} J^{-1}\left(J x_{n}-\left(\mu_{X}\right)^{-2} J\left(x_{n}-T x_{n}\right)\right)
$$

and

$$
y_{n+1}=Q_{C} J^{-1}\left(\alpha_{n} J y_{1}+\left(1-\alpha_{n}\right)\left(J y_{n}-\left(\mu_{X}\right)^{-2} J\left(y_{n}-T y_{n}\right)\right)\right)
$$

for all $n \in \mathbb{N}$, where $x_{1}, y_{1} \in C, \mu_{X}$ denotes the uniform convexity constant of $X, Q_{C}$ denotes the generalized projection of $X$ onto $C$, and $\left\{\alpha_{n}\right\}$ is a sequence of $[0,1]$. If $X$ is a Hilbert space, then (1.1) and (1.2) are reduced to

$$
x_{n+1}=T x_{n} \quad \text { and } \quad y_{n+1}=\alpha_{n} y_{1}+\left(1-\alpha_{n}\right) T y_{n}
$$

for all $n \in \mathbb{N}$, respectively.

This paper is organized as follows: In Section 2, we give some definitions and state some known results. In Section 3, we obtain two lemmas for a single firmly nonexpansive-like mapping. In Section 4, we construct strongly relatively nonexpansive sequences of mappings from a given sequence of firmly nonexpansive-like mappings. Using these results, we deduce two convergence theorems. In Section 5, we discuss some applications of our results.

\section{Preliminaries}

Throughout the present paper, we denote by $\mathbb{N}$ the set of all positive integers, $\mathbb{R}$ the set of all real numbers, $X$ a real Banach space with dual $X^{*},\|\cdot\|$ the norms of $X$ and $X^{*},\left\langle x, x^{*}\right\rangle$ the value of $x^{*} \in X^{*}$ at $x \in X, x_{n} \rightarrow x$ strong convergence of a sequence $\left\{x_{n}\right\}$ of $X$ to $x \in X$, $x_{n} \rightarrow x$ weak convergence of a sequence $\left\{x_{n}\right\}$ of $X$ to $x \in X, S_{X}$ the unit sphere of $X$, and $B_{X}$ the closed unit ball of $X$.

The normalized duality mapping of $X$ into $X^{*}$ is defined by

$$
J x=\left\{x^{*} \in X^{*}:\left\langle x, x^{*}\right\rangle=\|x\|^{2}=\left\|x^{*}\right\|^{2}\right\}
$$

for all $x \in X$. The space $X$ is said to be smooth if

$$
\lim _{t \rightarrow 0} \frac{\|x+t y\|-\|x\|}{t}
$$

exists for all $x, y \in S_{X}$. The space $X$ is also said to be uniformly smooth if (2.2) converges uniformly in $x, y \in S_{X}$. It is said to be strictly convex if $\|(x+y) / 2\|<1$ whenever $x, y \in S_{X}$ and $x \neq y$. It is said to be uniformly convex if $\delta_{X}(\varepsilon)>0$ for all $\varepsilon \in(0,2]$, where $\delta_{X}$ is the modulus of convexity of $X$ defined by

$$
\delta_{X}(\varepsilon)=\inf \left\{1-\left\|\frac{x+y}{2}\right\|: x, y \in B_{X},\|x-y\| \geq \varepsilon\right\}
$$

for all $\varepsilon \in[0,2]$. The space $X$ is said to be 2-uniformly convex if there exists $c>0$ such that $\delta_{X}(\varepsilon) \geq c \varepsilon^{2}$ for all $\varepsilon \in[0,2]$. It is obvious that every 2-uniformly convex Banach 
space is uniformly convex. It is known that all Hilbert spaces are uniformly smooth and 2uniformly convex. It is also known that all the Lebesgue spaces $L^{p}$ are uniformly smooth and 2-uniformly convex whenever $1<p \leq 2$; see [8,pp.198-203]. For a smooth Banach space, $J$ is said to be weakly sequentially continuous if $\left\{J x_{n}\right\}$ converges weakly* to $J x$ whenever $\left\{x_{n}\right\}$ is a sequence of $X$ such that $x_{n} \rightarrow x \in X$. We know the following fundamental result.

Lemma 2.1 ([8-10]) The space $X$ is 2-uniformly convex if and only if there exists $\mu \geq 1$ such that

$$
\frac{\|x+y\|^{2}+\|x-y\|^{2}}{2} \geq\|x\|^{2}+\left\|\mu^{-1} y\right\|^{2}
$$

for all $x, y \in X$.

The minimum value of the set of all $\mu \geq 1$ satisfying (2.4) for all $x, y \in X$ is denoted by $\mu_{X}$ and is called the 2-uniform convexity constant of $X$; see [9]. It is obvious that $\mu_{X}=1$ whenever $X$ is a Hilbert space.

In what follows throughout this section, we assume the following:

- $X$ is a smooth, strictly convex, and reflexive Banach space;

- $C$ is a nonempty closed convex subset of $X$.

In this case, $J$ is single valued, one to one, and onto; see $[11,12]$ for more details. We denote by $\phi$ the function of $X \times X$ into $\mathbb{R}$ defined by

$$
\phi(x, y)=\|x\|^{2}-2\langle x, J y\rangle+\|y\|^{2}
$$

for all $x, y \in X$; see $[13,14]$. It is known that

$$
\phi(x, y)=\phi(x, z)+\phi(z, y)+2\langle x-z, J z-J y\rangle
$$

for all $x, y, z \in X$. Using Lemma 2.1, we can show the following lemma.

Lemma 2.2 Suppose that $X$ is 2-uniformly convex. Then

$$
\left(\frac{1}{\mu_{X}}\|x-y\|\right)^{2} \leq \phi(x, y)
$$

for all $x, y \in X$.

Proof By (2.4) and the definition of $\mu_{X}$, we have

$$
\left\|\frac{u+v}{2}\right\|^{2} \leq \frac{\|u\|^{2}+\|v\|^{2}}{2}-\left(\frac{1}{2 \mu_{X}}\right)^{2}\|u-v\|^{2}
$$

for all $u, v \in X$. Let $x, y \in X$ be given. By (2.8) and induction, we can easily show that

$$
\begin{aligned}
& \left\|\left(1-\frac{1}{2^{n}}\right) y+\frac{1}{2^{n}} x\right\|^{2} \\
& \quad \leq\left(1-\frac{1}{2^{n}}\right)\|y\|^{2}+\frac{1}{2^{n}}\|x\|^{2}-\left(\frac{1}{\mu_{X}}\right)^{2}\left(1-\frac{1}{2^{n}}\right) \frac{1}{2^{n}}\|y-x\|^{2}
\end{aligned}
$$


for all $n \in \mathbb{N}$. Hence we have

$$
\begin{aligned}
& 2^{n}\left(\left\|y+\frac{1}{2^{n}}(x-y)\right\|^{2}-\|y\|^{2}\right) \\
& \quad \leq-\|y\|^{2}+\|x\|^{2}-\left(\frac{1}{\mu_{X}}\right)^{2}\left(1-\frac{1}{2^{n}}\right)\|y-x\|^{2}
\end{aligned}
$$

for all $n \in \mathbb{N}$. The smoothness of $X$ implies that

$$
2\langle x-y, J y\rangle=\lim _{t \rightarrow 0} \frac{\|y+t(x-y)\|^{2}-\|y\|^{2}}{t} .
$$

By (2.10) and (2.11), we have

$$
\begin{aligned}
2\langle x-y, J y\rangle & =\lim _{n \rightarrow \infty} 2^{n}\left(\left\|y+\frac{1}{2^{n}}(x-y)\right\|^{2}-\|y\|^{2}\right) \\
& \leq-\|y\|^{2}+\|x\|^{2}-\left(\frac{1}{\mu_{X}}\right)^{2}\|x-y\|^{2} .
\end{aligned}
$$

Therefore, we obtain $\left(\|x-y\| / \mu_{X}\right)^{2} \leq \phi(x, y)$ as desired.

The metric projection $P_{C}$ of $X$ onto $C$ and the generalized projection $Q_{C}$ of $X$ onto $C$ are defined by

$$
P_{C} x=\underset{y \in C}{\operatorname{argmin}}\|y-x\| \quad \text { and } \quad Q_{C} x=\underset{y \in C}{\operatorname{argmin}} \phi(y, x)
$$

for all $x \in X$, respectively. The following holds for $x \in X$ and $z \in C$ :

$$
z=P_{C} x \quad \Longleftrightarrow\langle y-z, J(x-z)\rangle \leq 0 \quad(\forall y \in C) ;
$$

see [12, Corollary 6.5.5]. The following also holds for $x \in X$ and $z \in C$ :

$$
z=Q_{C} x \quad \Longleftrightarrow \quad\langle y-z, J x-J z\rangle \leq 0 \quad(\forall y \in C) ;
$$

see [13, Remark 7.3] and [14, Proposition 4].

A mapping $T: C \rightarrow X$ is said to be a firmly nonexpansive-like mapping (or a mapping of type (P)) [1] if

$$
\langle T x-T y, J(x-T x)-J(y-T y)\rangle \geq 0
$$

for all $x, y \in C$; see also [2,3]. The set of all fixed points of $T$ is denoted by $\mathrm{F}(T)$. If $X$ is a Hilbert space, then $T$ is firmly nonexpansive-like if and only if it is firmly nonexpansive, i.e., $\|T x-T y\|^{2} \leq\langle T x-T y, x-y\rangle$ for all $x, y \in C$. It is known [1] that the following hold:

- the metric projection $P_{C}$ of $X$ onto $C$ is a firmly nonexpansive-like mapping and $\mathrm{F}\left(P_{C}\right)=C$

- if $A: X \rightarrow 2^{X^{*}}$ is maximal monotone and $\lambda>0$, then the resolvent $K_{\lambda}: X \rightarrow X$ of $A$ defined by $K_{\lambda}=\left(I+\lambda J^{-1} A\right)^{-1}$ is a firmly nonexpansive-like mapping and $\mathrm{F}\left(K_{\lambda}\right)=A^{-1} 0$. 
Let $T: C \rightarrow X$ be a mapping. A point $p \in C$ is said to be an asymptotic fixed point of $T$ if there exists a sequence $\left\{x_{n}\right\}$ of $C$ such that $x_{n} \rightarrow p$ and $x_{n}-T x_{n} \rightarrow 0$; see $[15,16]$. The set of all asymptotic fixed points of $T$ is denoted by $\hat{\mathrm{F}}(T)$. The mapping $T$ is said to be of type (r) if $\mathrm{F}(T)$ is nonempty and $\phi(u, T x) \leq \phi(u, x)$ for all $u \in \mathrm{F}(T)$ and $x \in C$. It is known that if $T$ is of type (r), then $\mathrm{F}(T)$ is closed and convex; see [16, Proposition 2.4]. The mapping $T$ is said to be of type (sr) if $T$ is of type (r) and $\phi\left(T z_{n}, z_{n}\right) \rightarrow 0$ whenever $\left\{z_{n}\right\}$ is a bounded sequence of $C$ such that $\phi\left(u, z_{n}\right)-\phi\left(u, T z_{n}\right) \rightarrow 0$ for some $u \in \mathrm{F}(T)$; see [17]. We know the following results:

Lemma 2.3 ([3, Lemma 2.2]) If $T: C \rightarrow X$ is a firmly nonexpansive-like mapping, then $\mathrm{F}(T)$ is a closed convex subset of $X$ and $\hat{\mathrm{F}}(T)=\mathrm{F}(T)$.

Lemma 2.4 ([17, Lemmas 3.2 and 3.3]) Suppose that $X$ is uniformly convex. If $S: X \rightarrow X$ and $T: C \rightarrow X$ are mappings of type $(r)$ such that $\mathrm{F}(S) \cap \mathrm{F}(T)$ is nonempty and $S$ or $T$ is of type (sr), then $S T: C \rightarrow X$ is of type $(r)$ and $\mathrm{F}(S T)=\mathrm{F}(S) \cap \mathrm{F}(T)$. Further, if both $S$ and $T$ are of type (sr), then so is $S T$.

Let $\left\{T_{n}\right\}$ be a sequence of mappings of $C$ into $X$. The set of all common fixed points of $\left\{T_{n}\right\}$ is denoted by $\mathrm{F}\left(\left\{T_{n}\right\}\right)$. The sequence $\left\{T_{n}\right\}$ is said to be of type (sr) (or strongly relatively nonexpansive) if $\mathrm{F}\left(\left\{T_{n}\right\}\right)$ is nonempty, each $T_{n}$ is of type (r), and $\phi\left(T_{n} z_{n}, z_{n}\right) \rightarrow 0$ whenever $\left\{z_{n}\right\}$ is a bounded sequence of $C$ such that $\phi\left(u, z_{n}\right)-\phi\left(u, T_{n} z_{n}\right) \rightarrow 0$ for some $u \in \mathrm{F}\left(\left\{T_{n}\right\}\right)$; see [18]. The sequence $\left\{T_{n}\right\}$ is said to satisfy the condition (Z) if every weak subsequential limit of $\left\{x_{n}\right\}$ belongs to $\mathrm{F}\left(\left\{T_{n}\right\}\right)$ whenever $\left\{x_{n}\right\}$ is a bounded sequence of $C$ such that $x_{n}-T_{n} x_{n} \rightarrow 0$; see [18].

Remark 2.5 For a mapping $T$ of $C$ into $X$, the following hold: $T$ is of type (sr) if and only if $\{T, T, \ldots\}$ is of type (sr); $\hat{\mathrm{F}}(T)=\mathrm{F}(T)$ if and only if $\{T, T, \ldots\}$ satisfies the condition (Z).

We know the following fundamental results; see [18, Theorem 3.4] for (i) and [19, Propositions 3 and 6] for (ii).

Lemma 2.6 Suppose that $X$ is uniformly convex. Let $\left\{S_{n}\right\}$ be a sequence of mappings of $X$ into itself and $\left\{T_{n}\right\}$ a sequence of mappings of $C$ into $X$ such that $\mathrm{F}\left(\left\{S_{n}\right\}\right) \cap \mathrm{F}\left(\left\{T_{n}\right\}\right)$ is nonempty, both $\left\{S_{n}\right\}$ and $\left\{T_{n}\right\}$ are of type (sr), and $S_{n}$ or $T_{n}$ is of type (sr) for all $n \in \mathbb{N}$. Then the following hold:

(i) $\left\{S_{n} T_{n}\right\}$ is of type (sr);

(ii) if $X$ is uniformly smooth and both $\left\{S_{n}\right\}$ and $\left\{T_{n}\right\}$ satisfy the condition (Z), then so does $\left\{S_{n} T_{n}\right\}$.

We know the following result; see [18, Theorem 4.1] for (i) and [20, Theorem 4.1] for (ii).

Theorem 2.7 Let $X$ be a smooth and uniformly convex Banach space, C a nonempty closed convex subset of $X$, and $\left\{T_{n}\right\}$ a sequence of mappings of $C$ into $X$ such that $\left\{T_{n}\right\}$ is of type $\left(\right.$ sr) and $\left\{T_{n}\right\}$ satisfies the condition (Z). Then the following hold:

(i) if $T_{n}(C) \subset C$ for all $n \in \mathbb{N}$ and $J$ is weakly sequentially continuous, then the sequence $\left\{x_{n}\right\}$ defined by $x_{1} \in C$ and $x_{n+1}=T_{n} x_{n}$ for all $n \in \mathbb{N}$ converges weakly to the strong limit of $\left\{Q_{F} x_{n}\right\}$; 
(ii) if $u$ is an element of $X$ and $\left\{\alpha_{n}\right\}$ is a sequence of $[0,1]$ such that $\alpha_{n}>0$ for all $n \in \mathbb{N}$, $\alpha_{n} \rightarrow 0$, and $\sum_{n=1}^{\infty} \alpha_{n}=\infty$, then the sequence $\left\{y_{n}\right\}$ defined by $y_{1} \in C$ and $y_{n+1}=Q_{C} J^{-1}\left(\alpha_{n} J u+\left(1-\alpha_{n}\right) J T_{n} y_{n}\right)$ for all $n \in \mathbb{N}$ converges strongly to $Q_{F} u$.

\section{Lemmas}

Throughout this section, we assume the following:

- $C$ is a nonempty closed convex subset of a smooth, strictly convex, and reflexive Banach space $X$;

- $T$ is a firmly nonexpansive-like mapping of $C$ into $X$;

- $S$ is a mapping of $C$ into $X$ defined by $S=J^{-1}(J-\beta J(I-T))$, where $\beta>0$ and $I$ denotes the identity mapping on $C$.

Lemma 3.1 The following hold:

(i) $\mathrm{F}(S)=\mathrm{F}(T)$ and $\mathrm{F}\left(Q_{C} S\right)=\mathrm{F}\left(P_{C} T\right)$;

(ii) if $\mathrm{F}(T)$ is nonempty, then $\mathrm{F}\left(P_{C} T\right)=\mathrm{F}(T)$.

Proof We can easily see that $\mathrm{F}(S)=\mathrm{F}(T)$. We first show that $\mathrm{F}\left(Q_{C} S\right)=\mathrm{F}\left(P_{C} T\right)$. Let $u \in C$ be given. Then it follows from (2.14) and (2.15) that

$$
\begin{aligned}
Q_{C}(S u)=u & \Longleftrightarrow\langle y-u, J S u-J u\rangle \leq 0 \quad(\forall y \in C) \\
& \Longleftrightarrow\langle y-u,-\beta J(u-T u)\rangle \leq 0 \quad(\forall y \in C) \\
& \Longleftrightarrow\langle y-u, J(T u-u)\rangle \leq 0 \quad(\forall y \in C) \\
& \Longleftrightarrow P_{C}(T u)=u .
\end{aligned}
$$

Thus we have $\mathrm{F}\left(Q_{C} S\right)=\mathrm{F}\left(P_{C} T\right)$.

We next show (ii). Suppose that $\mathrm{F}(T)$ is nonempty. It is sufficient to show that $\mathrm{F}\left(P_{C} T\right) \subset$ $\mathrm{F}(T)$. Let $v \in \mathrm{F}\left(P_{C} T\right)$ be given and fix $p \in \mathrm{F}(T)$. Then it follows from (2.14) that

$$
\langle p-v, J(T v-v)\rangle \leq 0
$$

On the other hand, since $T$ is firmly nonexpansive-like and $p \in \mathrm{F}(T)$, we know that

$$
\langle T v-p, J(T v-v)\rangle \leq 0
$$

By (3.2) and (3.3), we obtain $\|T v-v\|^{2} \leq 0$. Thus we know that $v \in \mathrm{F}(T)$.

Lemma 3.2 Suppose that $X$ is 2-uniformly convex and $\mathrm{F}(T)$ is nonempty. Then

$$
\phi(u, S x)+\frac{1}{2}\left(\frac{2}{\mu_{X}^{2}}-\beta\right)\|S x-x\|^{2} \leq \phi(u, x)
$$

for all $u \in \mathrm{F}(S)$ and $x \in C$.

Proof Let $u \in \mathrm{F}(S)$ and $x \in C$ be given. Then it follows from (2.6) and the definition of $S$ that

$$
\begin{aligned}
\phi(u, S x)+\phi(S x, x)-\phi(u, x) & =2\langle u-S x, J x-J S x\rangle \\
& =2 \beta\langle u-S x, J(x-T x)\rangle .
\end{aligned}
$$


Since $T$ is firmly nonexpansive-like and $u \in \mathrm{F}(T)$ by (i) of Lemma 3.1, we know that

$$
\begin{aligned}
\langle u-S x, J(x-T x)\rangle & =\langle u-T x, J(x-T x)\rangle+\langle T x-S x, J(x-T x)\rangle \\
& \leq\langle T x-S x, J(x-T x)\rangle .
\end{aligned}
$$

On the other hand, we have

$$
\begin{aligned}
& \langle T x-S x, J(x-T x)\rangle \\
& \quad=-\|T x-x\|^{2}+\langle x-S x, J(x-T x)\rangle \\
& \leq-\left(\|T x-x\|^{2}-\|x-S x\|\|x-T x\|\right) \\
& \quad=-\left(\|T x-x\|-\frac{1}{2}\|S x-x\|\right)^{2}+\frac{1}{4}\|S x-x\|^{2} \leq \frac{1}{4}\|S x-x\|^{2} .
\end{aligned}
$$

Since $\beta>0$, it follows from (3.5), (3.6), and (3.7) that

$$
\phi(u, S x)+\phi(S x, x)-\phi(u, x) \leq \frac{\beta}{2}\|S x-x\|^{2} .
$$

Since $X$ is 2 -uniformly convex, Lemma 2.2 implies that

$$
\left(\mu_{X}\right)^{-2}\|S x-x\|^{2} \leq \phi(S x, x)
$$

By (3.8) and (3.9), we obtain the desired inequality.

\section{Construction of strongly relatively nonexpansive sequences}

Throughout this section, we assume the following:

- $C$ is a nonempty closed convex subset of a smooth and 2-uniformly convex Banach space $X$

- $\left\{T_{n}\right\}$ is a sequence of firmly nonexpansive-like mappings of $C$ into $X$ such that $F=\mathrm{F}\left(\left\{T_{n}\right\}\right)$ is nonempty;

- $\left\{S_{n}\right\}$ is a sequence of mappings of $C$ into $X$ defined by

$$
S_{n}=J^{-1}\left(J-\beta_{n} J\left(I-T_{n}\right)\right)
$$

for all $n \in \mathbb{N}$, where $\left\{\beta_{n}\right\}$ is a sequence of real numbers such that $0<\inf _{n} \beta_{n}$ and $\sup _{n} \beta_{n}<2\left(\mu_{X}\right)^{-2}$ and $I$ denotes the identity mapping on $C$.

\section{Theorem 4.1 The following hold:}

(i) $\mathrm{F}\left(\left\{S_{n}\right\}\right)=F$ and $\left\{S_{n}\right\}$ is of type $(s r)$;

(ii) if $X$ is uniformly smooth and $\left\{T_{n}\right\}$ satisfies the condition (Z), then so does $\left\{S_{n}\right\}$.

Proof By (i) of Lemma 3.1, we know that $\mathrm{F}\left(\left\{S_{n}\right\}\right)=F$. We first show that $\left\{S_{n}\right\}$ is of type (sr). Note that $\mathrm{F}\left(\left\{S_{n}\right\}\right)$ is nonempty. By Lemma 3.2, we also know that each $S_{n}$ is a mapping of type (r) of $C$ into $X$. Suppose that $\left\{z_{n}\right\}$ is a bounded sequence of $C$ such that

$$
\phi\left(u, z_{n}\right)-\phi\left(u, S_{n} z_{n}\right) \rightarrow 0
$$


for some $u \in \mathrm{F}\left(\left\{S_{n}\right\}\right)$. Then it follows from Lemma 3.2 that

$$
0 \leq \frac{1}{2}\left(\frac{2}{\mu_{X}^{2}}-\beta_{n}\right)\left\|S_{n} z_{n}-z_{n}\right\|^{2} \leq \phi\left(u, z_{n}\right)-\phi\left(u, S_{n} z_{n}\right) \rightarrow 0 .
$$

Thus it follows from $\sup _{n} \beta_{n}<2\left(\mu_{X}\right)^{-2}$ that $\left\|S_{n} z_{n}-z_{n}\right\| \rightarrow 0$. Consequently, we have $\phi\left(S_{n} z_{n}, z_{n}\right) \rightarrow 0$ and hence $\left\{S_{n}\right\}$ is of type (sr).

We next show (ii). Suppose that $X$ is uniformly smooth and $\left\{T_{n}\right\}$ satisfies the condition (Z). Let $p$ be a weak subsequential limit of a bounded sequence $\left\{x_{n}\right\}$ of $C$ such that $x_{n}-S_{n} x_{n} \rightarrow 0$. By the definition of $S_{n}$, we have

$$
J\left(x_{n}-T_{n} x_{n}\right)=\frac{1}{\beta_{n}}\left(J x_{n}-J S_{n} x_{n}\right)
$$

for all $n \in \mathbb{N}$. Since $J$ is uniformly norm-to-norm continuous on each nonempty bounded subset of $X$ and $\sup _{n} 1 / \beta_{n}<\infty$, it follows from (4.4) that

$$
\left\|x_{n}-T_{n} x_{n}\right\|=\frac{1}{\beta_{n}}\left\|J x_{n}-J S_{n} x_{n}\right\| \rightarrow 0
$$

By assumption, we know that $p \in F=\mathrm{F}\left(\left\{S_{n}\right\}\right)$. Therefore, $\left\{S_{n}\right\}$ satisfies the condition (Z).

By Lemma 2.3, Remark 2.5, and Theorem 4.1, we obtain the following.

Corollary 4.2 Let $T$ be a firmly nonexpansive-like mapping of $C$ into $X$ such that $\mathrm{F}(T)$ is nonempty and $S$ a mapping of $C$ into $X$ defined by

$$
S=J^{-1}(J-\beta J(I-T))
$$

where $0<\beta<2\left(\mu_{X}\right)^{-2}$. Then the following hold:

(i) $\mathrm{F}(S)=\mathrm{F}(T)$ and $S$ is of type (sr);

(ii) if $X$ is uniformly smooth, then $\hat{\mathrm{F}}(S)=\mathrm{F}(S)$.

We next show one of our main results in the present paper.

Theorem 4.3 Let $\left\{U_{n}\right\}$ be a sequence of mappings of $C$ into itself defined by

$$
U_{n}=Q_{C} S_{n}
$$

for all $n \in \mathbb{N}$. Then the following hold:

(i) $\mathrm{F}\left(\left\{U_{n}\right\}\right)=F$ and $\left\{U_{n}\right\}$ is of type $($ sr);

(ii) if $X$ is uniformly smooth and $\left\{T_{n}\right\}$ satisfies the condition $(Z)$, then so does $\left\{U_{n}\right\}$.

Proof By Lemma 3.1, we know that $\mathrm{F}\left(S_{n}\right)=\mathrm{F}\left(T_{n}\right)=\mathrm{F}\left(U_{n}\right)$ for all $n \in \mathbb{N}$ and hence $\mathrm{F}\left(\left\{U_{n}\right\}\right)=$ $F \neq \emptyset$. We first show that $\left\{U_{n}\right\}$ is of type (sr). By (i) of Corollary 4.2, we know that each $S_{n}$ is of type (sr). Since $Q_{C}$ is of type (sr) of $X$ into itself and

$$
\mathrm{F}\left(Q_{C}\right) \cap \mathrm{F}\left(S_{n}\right)=\mathrm{F}\left(T_{n}\right) \supset F \neq \emptyset,
$$


Lemma 2.4 implies that each $U_{n}=Q_{C} S_{n}$ is also of type (sr). Since $\left\{Q_{C}, Q_{C}, \ldots\right\}$ is of type (sr) by Remark 2.5, $\left\{S_{n}\right\}$ is of type (sr) by (i) of Theorem 4.1, and

$$
\mathrm{F}\left(Q_{C}\right) \cap \mathrm{F}\left(\left\{S_{n}\right\}\right)=F \neq \emptyset,
$$

the part (i) of Lemma 2.6 implies that $\left\{U_{n}\right\}$ is of type (sr).

We finally show (ii). Suppose that $X$ is uniformly smooth and $\left\{T_{n}\right\}$ satisfies the condition $(Z)$. Since $C$ is weakly closed, we can easily see that $\hat{\mathrm{F}}\left(Q_{C}\right)=\mathrm{F}\left(Q_{C}\right)=C$. This implies that $\left\{Q_{C}, Q_{C}, \ldots\right\}$ satisfies the condition (Z). By (ii) of Theorem 4.1, we know that $\left\{S_{n}\right\}$ satisfies the condition (Z). Thus (ii) of Lemma 2.6 implies the conclusion.

By Lemma 2.3, Remark 2.5, and Theorem 4.3, we obtain the following.

Corollary 4.4 Let $T$ be a firmly nonexpansive-like mapping of $C$ into $X$ such that $\mathrm{F}(T)$ is nonempty and $U$ a mapping of $C$ into itself defined by

$$
U=Q_{C} J^{-1}(J-\beta J(I-T))
$$

where $0<\beta<2\left(\mu_{X}\right)^{-2}$. Then the following hold:

(i) $\mathrm{F}(U)=\mathrm{F}(T)$ and $U$ is of type (sr);

(ii) if $X$ is uniformly smooth, then $\hat{\mathrm{F}}(U)=\mathrm{F}(U)$.

As a direct consequence of (i) of Theorem 2.7 and Theorem 4.3, we obtain the following result.

Theorem 4.5 Let $X$ be a uniformly smooth and 2-uniformly convex Banach space, $C$ a nonempty closed convex subset of $X,\left\{T_{n}\right\}$ a sequence of firmly nonexpansive-like mappings of $C$ into $X$ such that $F=F\left(\left\{T_{n}\right\}\right)$ is nonempty and $\left\{T_{n}\right\}$ satisfies the condition $(\mathrm{Z}),\left\{\beta_{n}\right\}$ a sequence of real numbers such that

$$
0<\inf _{n} \beta_{n} \text { and } \sup _{n} \beta_{n}<\frac{2}{\left(\mu_{X}\right)^{2}},
$$

and $\left\{x_{n}\right\}$ a sequence defined by $x_{1} \in C$ and

$$
x_{n+1}=Q_{C} J^{-1}\left(J x_{n}-\beta_{n} J\left(x_{n}-T_{n} x_{n}\right)\right)
$$

for all $n \in \mathbb{N}$. IfJ is weakly sequentially continuous, then $\left\{x_{n}\right\}$ converges weakly to the strong limit of $\left\{Q_{F} x_{n}\right\}$.

As a direct consequence of (ii) of Theorem 2.7 and Theorem 4.1, we obtain the following result.

Theorem 4.6 Let $X, C,\left\{T_{n}\right\}, F,\left\{\beta_{n}\right\}$ be the same as in Theorem 4.5, $\left\{\alpha_{n}\right\}$ a sequence of $[0,1]$ such that $\alpha_{n}>0$ for all $n \in \mathbb{N}, \alpha_{n} \rightarrow 0$, and $\sum_{n=1}^{\infty} \alpha_{n}=\infty$, u an element of $X$, and $\left\{y_{n}\right\}$ a sequence defined by $y_{1} \in C$ and

$$
y_{n+1}=Q_{C} J^{-1}\left(\alpha_{n} J u+\left(1-\alpha_{n}\right)\left(J y_{n}-\beta_{n} J\left(y_{n}-T_{n} y_{n}\right)\right)\right)
$$

for all $n \in \mathbb{N}$. Then $\left\{y_{n}\right\}$ converges strongly to $Q_{F} u$. 
By Lemma 2.3, Theorem 4.5, and Theorem 4.6, we obtain the following corollary for a single firmly nonexpansive-like mapping.

Corollary 4.7 Let $X$ be a uniformly smooth and 2-uniformly convex Banach space, $C$ a nonempty closed convex subset of $X$, and $T$ a firmly nonexpansive-like mapping of $C$ into $X$ such that $\mathrm{F}(T)$ is nonempty. Then the following hold:

(i) if $J$ is weakly sequentially continuous, then the sequence $\left\{x_{n}\right\}$ defined by $x_{1} \in C$ and (1.1) for all $n \in \mathbb{N}$ converges weakly to the strong limit of $\left\{Q_{\mathrm{F}(T)} x_{n}\right\}$;

(ii) if $\left\{\alpha_{n}\right\}$ is a sequence of $[0,1]$ such that $\alpha_{n}>0$ for all $n \in \mathbb{N}, \alpha_{n} \rightarrow 0$, and $\sum_{n=1}^{\infty} \alpha_{n}=\infty$, then the sequence $\left\{y_{n}\right\}$ defined by $y_{1} \in C$ and (1.2) for all $n \in \mathbb{N}$ converges strongly to $Q_{\mathrm{F}(T)} u$.

Remark 4.8 Since $\mu_{X}=1$ and $J$ is the identity mapping on $C$ in the case when $X$ is a Hilbert space, the part (i) of Corollary 4.7 is a generalization of Martinet's theorem [4, Théorème 1].

\section{Applications}

Using Theorem 4.5, we first study the problem of approximating zero points of maximal monotone operators.

Corollary 5.1 Let $X$ be a uniformly smooth and 2-uniformly convex Banach space, $A: X \rightarrow 2^{X^{*}}$ a maximal monotone operator such that $F=A^{-1} 0$ is nonempty, $\left\{\lambda_{n}\right\}$ and $\left\{\beta_{n}\right\}$ sequences real numbers such that $0<\inf _{n} \lambda_{n}, 0<\inf _{n} \beta_{n}$, and $\sup _{n} \beta_{n}<2\left(\mu_{X}\right)^{-2},\left\{K_{\lambda_{n}}\right\}$ a sequence of mappings defined by $K_{\lambda_{n}}=\left(I+\lambda_{n} J^{-1} A\right)^{-1}$ for all $n \in \mathbb{N}$, where I denotes the identity mapping on $X$, and $\left\{x_{n}\right\}$ a sequence defined by $x_{1} \in X$ and

$$
x_{n+1}=J^{-1}\left(J x_{n}-\beta_{n} J\left(x_{n}-K_{\lambda_{n}} x_{n}\right)\right)
$$

for all $n \in \mathbb{N}$. IfJ is weakly sequentially continuous, then $\left\{x_{n}\right\}$ converges weakly to the strong limit of $\left\{Q_{F} x_{n}\right\}$.

Proof It is well known that each $K_{\lambda_{n}}$ is a single valued mapping of $X$ into itself and $\mathrm{F}\left(K_{\lambda_{n}}\right)=$ $F$; see $[21,22]$. We also know that each $K_{\lambda_{n}}$ is firmly nonexpansive-like and $\left\{K_{\lambda_{n}}\right\}$ satisfies the condition $(Z)$; see $[1,3]$. Therefore, Theorem 4.5 implies the conclusion.

Remark 5.2 Corollary 5.1 is a generalization of Rockafellar's weak convergence theorem [23] for the proximal point algorithm in Hilbert spaces.

Using Corollary 5.1, we next study the problem of minimizing a convex function. For a Banach space $X$ and a function $f: X \rightarrow(-\infty, \infty]$, we denote by $\partial f$ the subdifferential of $f$ defined by

$$
\partial f(x)=\left\{x^{*} \in X^{*}:\left(f-x^{*}\right)(x)=\inf \left(f-x^{*}\right)(X)\right\}
$$

for all $x \in X$.

Corollary 5.3 Let $X,\left\{\lambda_{n}\right\}$, and $\left\{\beta_{n}\right\}$ be the same as in Corollary 5.1, $f: X \rightarrow(-\infty, \infty] a$ proper lower semicontinuous convex function such that $F=\operatorname{argmin} f$ is nonempty, and $\left\{x_{n}\right\}$ 
a sequence defined by $x_{1} \in X$ and

$$
\left\{\begin{array}{l}
y_{n}=\operatorname{argmin}_{y \in X}\left\{f(y)+\left(2 \lambda_{n}\right)^{-1}\left\|y-x_{n}\right\|^{2}\right\} \\
x_{n+1}=J^{-1}\left(J x_{n}-\beta_{n} J\left(x_{n}-y_{n}\right)\right)
\end{array}\right.
$$

for all $n \in \mathbb{N}$. If $J$ is weakly sequentially continuous, then $\left\{x_{n}\right\}$ converges weakly to the strong limit of $\left\{Q_{F} x_{n}\right\}$.

Proof We know that $\partial f: X \rightarrow 2^{X^{*}}$ is maximal monotone $[24,25]$ and $(\partial f)^{-1}(0)=\operatorname{argmin} f$. We also know that

$$
\left(I+\lambda J^{-1} \partial f\right)^{-1} x=\underset{y \in X}{\operatorname{argmin}}\left\{f(y)+(2 \lambda)^{-1}\|y-x\|^{2}\right\}
$$

for all $\lambda>0$ and $x \in X$, where $I$ denotes the identity mapping on $X$. Therefore, the result follows from Corollary 5.1.

Using Theorem 4.6, we can similarly show the following corollary.

Corollary 5.4 Let $X, A, F,\left\{\lambda_{n}\right\},\left\{\beta_{n}\right\}$, and $\left\{K_{\lambda_{n}}\right\}$ be the same as in Corollary 5.1, $u$ an element of $X$, and $\left\{y_{n}\right\}$ a sequence defined by $y_{1} \in X$ and

$$
y_{n+1}=J^{-1}\left(\alpha_{n} J u+\left(1-\alpha_{n}\right)\left(J y_{n}-\beta_{n} J\left(y_{n}-K_{\lambda_{n}} y_{n}\right)\right)\right)
$$

for all $n \in \mathbb{N}$, where $\left\{\alpha_{n}\right\}$ is a sequence of $[0,1]$ such that $\alpha_{n}>0$ for all $n \in \mathbb{N}, \alpha_{n} \rightarrow 0$, and $\sum_{n=1}^{\infty} \alpha_{n}=\infty$. Then $\left\{y_{n}\right\}$ converges strongly to $Q_{F} u$.

Using the results obtained in Section 4 and (i) of Theorem 2.7, we next study the problem of approximating common points of a given family of closed convex sets.

Corollary 5.5 Let $X$ be a uniformly smooth and 2-uniformly convex Banach space, I the set $\{1,2, \ldots, m\}$, where $m$ is a positive integer, $\left\{C_{k}\right\}_{k \in \mathcal{I}}$ a finite family of closed convex subsets of $X$ such that $F=\bigcap_{k \in \mathcal{I}} C_{k}$ is nonempty, $\left\{\beta_{n, k}\right\}_{n \in \mathbb{N}, k \in \mathcal{I}}$ a sequence of real numbers such that $0<\inf _{n} \beta_{n, k}$ and $\sup _{n} \beta_{n, k}<2\left(\mu_{X}\right)^{-2}$ for all $k \in \mathcal{I},\left\{S_{n, k}\right\}_{n \in \mathbb{N}, k \in \mathcal{I}}$ a sequence of mappings defined by

$$
S_{n, k}=J^{-1}\left(J-\beta_{n, k} J\left(I-P_{C_{k}}\right)\right)
$$

for all $n \in \mathbb{N}$ and $k \in \mathcal{I}$, and $\left\{x_{n}\right\}$ a sequence defined by $x_{1} \in X$ and

$$
x_{n+1}=S_{n, 1} S_{n, 2} \cdots S_{n, m} x_{n}
$$

for all $n \in \mathbb{N}$. IfJ is weakly sequentially continuous, then $\left\{x_{n}\right\}$ converges weakly to the strong limit of $\left\{Q_{F} x_{n}\right\}$.

Proof For the sake of simplicity, we give the proof in the case when $\mathcal{I}=\{1,2,3\}$. Set

$$
U_{n}=S_{n, 1}, \quad V_{n}=S_{n, 2} \quad \text { and } \quad W_{n}=S_{n, 3}
$$


for all $n \in \mathbb{N}$. Note that $x_{n+1}=U_{n} V_{n} W_{n} x_{n}$ for all $n \in \mathbb{N}, P_{C_{k}}$ is firmly nonexpansive-like, and $\hat{\mathrm{F}}\left(P_{C_{k}}\right)=\mathrm{F}\left(P_{C_{k}}\right)=C_{k}$ for all $k \in\{1,2,3\}$. By Theorem 4.1 and Corollary 4.2, we know that the following hold:

- $\mathrm{F}\left(\left\{U_{n}\right\}\right)=C_{1}, \mathrm{~F}\left(\left\{V_{n}\right\}\right)=C_{2}$, and $\mathrm{F}\left(\left\{W_{n}\right\}\right)=C_{3}$;

- $U_{n}, V_{n}$, and $W_{n}$ are of type (sr) for all $n \in \mathbb{N}$;

- $\left\{U_{n}\right\},\left\{V_{n}\right\}$, and $\left\{W_{n}\right\}$ are of type (sr);

- $\left\{U_{n}\right\},\left\{V_{n}\right\}$, and $\left\{W_{n}\right\}$ satisfy the condition (Z).

Since

$$
\mathrm{F}\left(\left\{U_{n}\right\}\right) \cap \mathrm{F}\left(\left\{V_{n}\right\}\right)=C_{1} \cap C_{2} \supset F \neq \emptyset,
$$

Lemmas 2.4 and 2.6 ensure that the following hold:

- $\mathrm{F}\left(\left\{U_{n} V_{n}\right\}\right)=\mathrm{F}\left(\left\{U_{n}\right\}\right) \cap \mathrm{F}\left(\left\{V_{n}\right\}\right)=C_{1} \cap C_{2}$;

- each $U_{n} V_{n}$ is of type (sr);

- $\left\{U_{n} V_{n}\right\}$ is of type (sr) and satisfies the condition (Z).

Since

$$
\mathrm{F}\left(\left\{U_{n} V_{n}\right\}\right) \cap \mathrm{F}\left(\left\{W_{n}\right\}\right)=\left(C_{1} \cap C_{2}\right) \cap C_{3}=F \neq \emptyset,
$$

Lemmas 2.4 and 2.6 also ensure that $\mathrm{F}\left(\left\{U_{n} V_{n} W_{n}\right\}\right)=\mathrm{F}\left(\left\{U_{n} V_{n}\right\}\right) \cap \mathrm{F}\left(\left\{W_{n}\right\}\right)=F,\left\{U_{n} V_{n} W_{n}\right\}$ is of type (sr), and $\left\{U_{n} V_{n} W_{n}\right\}$ satisfies the condition (Z). Therefore, (i) of Theorem 2.7 implies the conclusion.

Using the results obtained in Section 4 and (ii) of Theorem 2.7, we can similarly show the following result.

Corollary 5.6 Let $X, \mathcal{I},\left\{C_{k}\right\}_{k=1}^{m}, F,\left\{\beta_{n, k}\right\}_{n \in \mathbb{N}, k \in \mathcal{I}},\left\{S_{n, k}\right\}_{n \in \mathbb{N}, k \in \mathcal{I}}$ be the same as in Corollary 5.5, $u$ an element of $X$, and $\left\{y_{n}\right\}$ a sequence defined by $y_{1} \in X$ and

$$
y_{n+1}=J^{-1}\left(\alpha_{n} J u+\left(1-\alpha_{n}\right) J S_{n, 1} S_{n, 2} \cdots S_{n, m} y_{n}\right)
$$

for all $n \in \mathbb{N}$, where $\left\{\alpha_{n}\right\}$ is a sequence of $[0,1]$ such that $\alpha_{n}>0$ for all $n \in \mathbb{N}, \alpha_{n} \rightarrow 0$, and $\sum_{n=1}^{\infty} \alpha_{n}=\infty$. Then $\left\{y_{n}\right\}$ converges strongly to $Q_{F} u$.

The authors declare that they have no competing interests.

Authors' contributions

All authors contributed equally to the writing of this paper. All authors read and approved the final manuscript.

\section{Author details}

${ }^{1}$ Department of Economics, Chiba University, Yayoi-cho, Inage-ku, Chiba-shi, Chiba, 263-8522, Japan. ${ }^{2}$ Department of

Computer Science and Intelligent Systems, Oita University, Dannoharu, Oita-shi, Oita, 870-1192, Japan.

\section{Acknowledgements}

The authors would like to express their sincere appreciation to the anonymous referees for their helpful comments on the original version of the manuscript. 


\section{References}

1. Aoyama, K, Kohsaka, F, Takahashi, W: Three generalizations of firmly nonexpansive mappings: their relations and continuity properties. J. Nonlinear Convex Anal. 10, 131-147 (2009)

2. Aoyama, K, Kohsaka, F: Existence of fixed points of firmly nonexpansive-like mappings in Banach spaces. Fixed Point Theory Appl. 2010, Article ID 512751 (2010)

3. Aoyama, K, Kohsaka, F, Takahashi, W: Strong convergence theorems for a family of mappings of type $(P)$ and applications. In: Nonlinear Analysis and Optimization, pp. 1-17. Yokohama Publishers, Yokohama (2009)

4. Martinet, B: Détermination approchée d'un point fixe d'une application pseudo-contractante. Cas de l'application prox. C. R. Acad. Sci. Paris, Sér. A-B 274, A163-A165 (1972)

5. Kimura, Y, Nakajo, K: The problem of image recovery by the metric projections in Banach spaces. Abstr. Appl. Anal. 2013, Article ID 817392 (2013)

6. Crombez, G: Image recovery by convex combinations of projections. J. Math. Anal. Appl. 155, 413-419 (1991)

7. Brègman, LM: The method of successive projection for finding a common point of convex sets. Sov. Math. Dokl. 6 , 688-692 (1965)

8. Beauzamy, B: Introduction to Banach Spaces and Their Geometry. North-Holland, Amsterdam (1985)

9. Ball, K, Carlen, EA, Lieb, EH: Sharp uniform convexity and smoothness inequalities for trace norms. Invent. Math. 115 463-482 (1994)

10. Takahashi, Y, Hashimoto, K, Kato, M: On sharp uniform convexity, smoothness, and strong type, cotype inequalities. J. Nonlinear Convex Anal. 3, 267-281 (2002)

11. Cioranescu, I: Geometry of Banach Spaces, Duality Mappings and Nonlinear Problems. Kluwer Academic, Dordrecht (1990)

12. Takahashi, W: Nonlinear Functional Analysis. Fixed Point Theory and Its Applications. Yokohama Publishers, Yokohama (2000)

13. Alber, Yl: Metric and generalized projection operators in Banach spaces: properties and applications. In: Theory and Applications of Nonlinear Operators of Accretive and Monotone Type, vol. 178. Dekker, New York (1996)

14. Kamimura, S, Takahashi, W: Strong convergence of a proximal-type algorithm in a Banach space. SIAM J. Optim. 13, 938-945 (2002)

15. Matsushita, S, Takahashi, W: Weak and strong convergence theorems for relatively nonexpansive mappings in Banach spaces. Fixed Point Theory Appl. 2004, 37-47 (2004)

16. Matsushita, S, Takahashi, W: A strong convergence theorem for relatively nonexpansive mappings in a Banach space. J. Approx. Theory 134, 257-266 (2005)

17. Aoyama, K, Kohsaka, F, Takahashi, W: Strong convergence theorems by shrinking and hybrid projection methods for relatively nonexpansive mappings in Banach spaces. In: Nonlinear Analysis and Convex Analysis, pp. 7-26. Yokohama Publishers, Yokohama (2009)

18. Aoyama, K, Kohsaka, F, Takahashi, W: Strongly relatively nonexpansive sequences in Banach spaces and applications. J. Fixed Point Theory Appl. 5, 201-224 (2009)

19. Aoyama, K: Asymptotic fixed points of sequences of quasi-nonexpansive type mappings. In: Banach and Function Spaces III, pp. 343-350. Yokohama Publishers, Yokohama (2011)

20. Aoyama, K, Kimura, Y, Kohsaka, F: Strong convergence theorems for strongly relatively nonexpansive sequences and applications. J. Nonlinear Anal. Optim. 3, 67-77 (2012)

21. Barbu, V: Nonlinear Semigroups and Differential Equations in Banach Spaces. Editura Academiei Republicii Socialiste România, Bucharest (1976)

22. Rockafellar, RT: On the maximality of sums of nonlinear monotone operators. Trans. Am. Math. Soc. 149, 75-88 (1970)

23. Rockafellar, RT: Monotone operators and the proximal point algorithm. SIAM J. Control Optim. 14, $877-898$ (1976)

24. Rockafellar, RT: Characterization of the subdifferentials of convex functions. Pac. J. Math. 17, 497-510 (1966)

25. Rockafellar, RT: On the maximal monotonicity of subdifferential mappings. Pac. J. Math. 33, 209-216 (1970)

10.1186/1687-1812-2014-95

Cite this article as: Aoyama and Kohsaka: Strongly relatively nonexpansive sequences generated by firmly nonexpansive-like mappings. Fixed Point Theory and Applications 2014, 2014:95

\section{Submit your manuscript to a SpringerOpen ${ }^{\odot}$ journal and benefit from:}

- Convenient online submission

- Rigorous peer review

Immediate publication on acceptance

- Open access: articles freely available online

- High visibility within the field

- Retaining the copyright to your article 